MAGNETOHYDRODYNAMICS Vol. 54 (2018), No. 1-2, pp. 103-107

DOI: $10.22364 / \mathrm{mhd} .54 .1-2.18$

\title{
INFLUENCE OF THE PARTICLE SHAPE ON THE EQUILIBRIUM MORPHOLOGIES OF SUPRACOLLOIDAL MAGNETIC FILAMENTS
}

\author{
E.S.Pyanzina ${ }^{1}$, E.V.Novak ${ }^{1}$, D.A. Rozhkov², \\ A.V. Gudkova ${ }^{1}$, P.A. Sánchez ${ }^{2}$ \\ 1 Ural Federal University, 51 Lenin ave., 620000 Ekaterinburg, Russia \\ ${ }^{2}$ University of Vienna, Sensengasse 8, 1090, Vienna, Austria
}

We investigate equilibrium morphologies of linear and ring-shaped magnetic filaments made from crosslinked ferromagnetic spherical or ellipsoidal colloidal particles. Using Langevin dynamics simulations, we calculate the radius of gyration and the total magnetic moment of a single filament at zero field and different temperatures, analyzing the influence of the particles shape, the strength of their magnetic moment and the filament length. Our results show that, among such parameters, the shape of the particles has the strongest qualitative impact on the equilibrium behavior of the filaments.

Introduction. Magnetic colloidal particles and materials based on them have been studied for already several decades. Conventional magnetic fluids, gels and elastomers are usually produced by means of relatively simple techniques. This provides a limited control of their microstructure and magnetic response. A novel and promising approach is the creation of small aggregates of magnetic particles with well defined shapes, permanently stabilized by polymer crosslinking in order to be operated as building blocks of more sophisticated magnetoresponsive materials. Using modern experimental techniques like DNA crosslinking [1] or DNA designed self-assembly [2], one can have the possibility to create rather flexible linear polymer-like chains of magnetic colloidal particles, also known as magnetic filaments. On the other hand, in recent years, magnetic anisotropic particles have become an independent and rapidly growing branch in dipolar soft matter research. The study of anisotropic magnetic particles addresses particles with internal anisotropies like capped colloids and particles with shifted dipoles [3] or Janus particles [4] and particles with shape anisotropies like magnetic rods or ellipsoids [5]. In this paper, for the first time we study magnetic filaments made of ellipsoidal magnetic particles by comparing their equilibrium behavior with the corresponding to filaments of spherical particles.

1. Magnetic filament model. To represent magnetic filaments, we employ a bead-spring model introduced in our previous works [6]. The magnetic beads carry a point magnetic dipole of moment $\mathbf{m}$ at their centers of geometry. The magnetic interactions are, therefore, calculated by means of the dipole-dipole pair potential:

$$
U_{d}(i j)=-\left[3 \frac{\left(\mathbf{m}_{i} \cdot \mathbf{r}_{i j}\right)\left(\mathbf{m}_{j} \cdot \mathbf{r}_{i j}\right)}{r_{i j}^{5}}-\frac{\left(\mathbf{m}_{i} \cdot \mathbf{m}_{j}\right)}{r_{i j}^{3}}\right],
$$

where $\mathbf{r}_{i j}$ is the displacement vector connecting the centers of geometry of the particles $i$ and $j$. We model the particle's shape anisotropy using an orientationdependent steric interaction. This is a modified Gay-Berne potential [7] that allows to represent ellipsoids with any arbitrary aspect ratio, $X_{0}=a / b$, where $a$ and $b$ are the main geometry axes of the body, so that $X_{0}=1$ for the sphere and 
$X_{0}>1$ for the ellipsoid. This potential has the form:

$U_{\mathrm{GB}}(i j)= \begin{cases}\varepsilon\left(\mathbf{u}_{i}, \mathbf{u}_{j}\right)\left[\left(\frac{\sigma_{0}}{r_{i j}-\sigma(\cdot)+\sigma_{0}}\right)^{12}-\left(\frac{\sigma_{0}}{r_{i j}-\sigma(\cdot)+\sigma_{0}}\right)^{6}+\frac{1}{4}\right], & r_{i j} \leq r_{\mathrm{c}} \\ 0, & r_{i j}>r_{\mathrm{c}}\end{cases}$

where $\mathbf{u}_{i(j)}$ is the unit vector along the axis $b$ of the particle $i(j), \sigma_{0}=2 \sqrt{2} a$, $\sigma(\cdot)=\sigma\left(\mathbf{u}_{i}, \mathbf{u}_{j}, \hat{\mathbf{r}}_{i j}\right)$ is the effective interparticle distance and $\varepsilon_{0}$ denotes the energy scale of the interaction. Finally, the permanent crosslinks between the particles are represented as a harmonic potential that connects adjacent particles along the chain. In the case of spherical particles, this bonding potential connects their surfaces [6], whereas for ellipsoids, it connects their centers of geometry. In spheres, the dipole moment remains oriented in the direction defined by the surface crosslinks, whereas in ellipsoids it is aligned parallel to their axis $a$. With this model, we performed Langevin dynamics simulations in the NVT ensemble by means of the ESPResSo simulation package [8]. More details of the simulation protocol and parameters are presented in $[6,9]$.

\section{Results and discussion.}

Here, we express all the physical parameters in a typical system of reduced units [9].

The first parameter which was calculated for the characterization of the equilibrium structures of the filaments is the radius of gyration defined in terms of the filament length $L$, the position of the center of mass of the filament $\mathbf{R}_{\mathrm{cm}}$ and the positions of each of its particles $\mathbf{r}_{i}$ as

$$
R_{\mathrm{g}}=\left[\frac{1}{L} \sum_{i=1}^{L}\left(\mathbf{r}_{i}-\mathbf{R}_{\mathrm{cm}}\right)^{2}\right]^{1 / 2} .
$$

Fig. 1 shows the results of $R_{\mathrm{g}}$ as a function of the temperature for the case $L=10$. For open filaments with spherical particles, we can see that $R_{\mathrm{g}}$ grows with

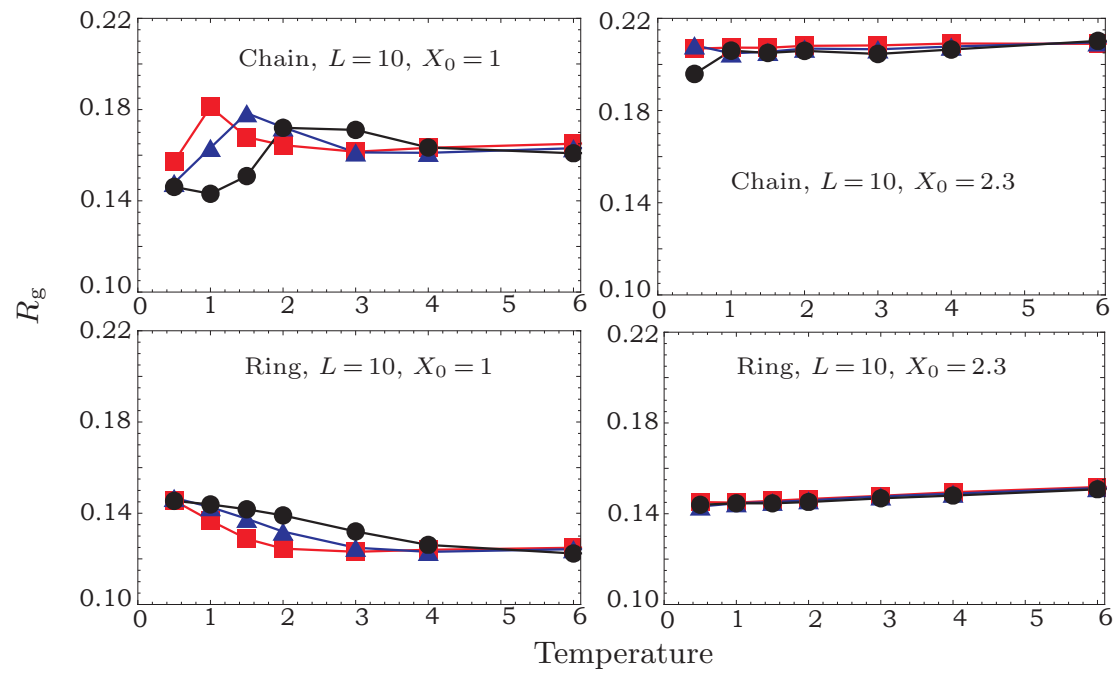

Fig. 1. The radius of gyration as a function of the temperature. First column: results for a filament composed of spheres $\left(X_{0}=1\right)$, second column: a filament composed of ellipsoids $\left(X_{0}=2.3\right)$. Different symbols correspond to different values of the magnetic moment: squares $-m^{2}=3$, triangles $-m^{2}=5$, dots $-m^{2}=8$. 
decreasing temperature until it experiences a drop, corresponding to the closure of the open chain into a ring (see snapshots in Fig. 3). As known, the temperature at which the closure takes place depends on both the magnetic moment of the particles and the filament length. Filaments with ellipsoids do not experience any closure and their $R_{\mathrm{g}}$ remains nearly constant within the sampled range of temperatures. This is due to the fact that ellipsoids can only form rings by connecting the filament free ends with their dipoles forming an antiparallel pair (see [10]), which is energetically much less favorable than the head-to-tail pair formed in the closure of spherical particles. The same qualitative difference was observed for rings: while $R_{\mathrm{g}}$ slightly decreases with the temperature for the case of spherical particles, the rings of ellipsoids, in turn, keep an almost constant value. This proves that the rings of spheres are more flexible than the rings of ellipsoids due to the geometrical constraints of the latter.

The second parameter analyzed is the relative net magnetic moment of the filament $M$ given by the normalized vector sum of the dipolar moments of its particles:

$$
M=\frac{1}{m L}\left|\sum_{i=1}^{L} \mathbf{m}_{i}\right| .
$$

Fig. 2 displays the results for $M$ obtained under the same conditions as discussed above. Here, the closure of the open filaments with spheres is much more clearly signaled as $M$ drops to almost zero for closed filaments. The rest of cases, i.e. open filaments with ellipsoids and both types of rings, show a monotonic decrease of $M$ at cooling, but the filaments of ellipsoids have values of $M$ significantly lower than their spherical counterparts. This is due to the alternating antiparallel orientation of the dipoles which they tend to keep independently on their open or closed arrangement, as the snapshots in Fig. 3 illustrate.

Finally, the results for longer filament lengths $(L=\{15,30\}$, not shown) exhibit the same qualitative behavior, with only moderate quantitative differences.

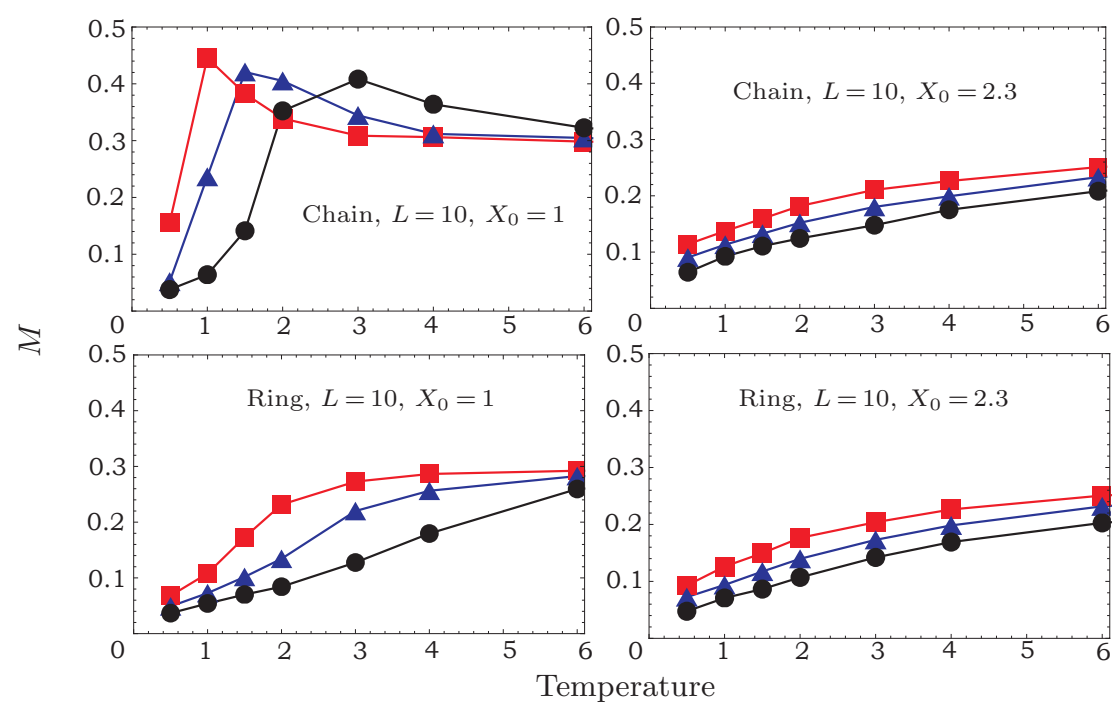

Fig. 2. The total magnetic moment of filaments as a function of the temperature. The same arrangement as in Fig. 1. 


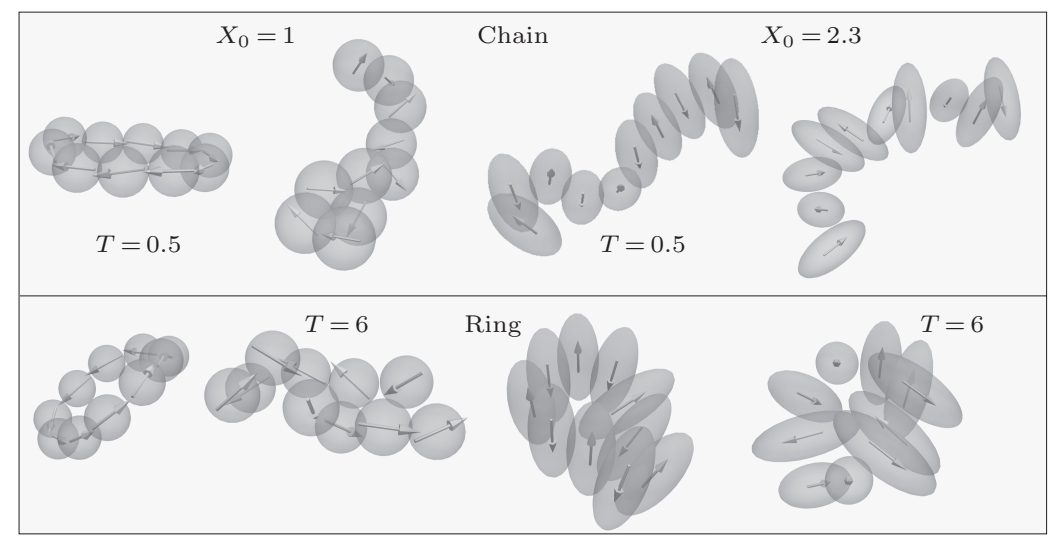

Fig. 3. Some typical configuration snapshots for different system parameters.

3. Conclusions. We studied for the first time the equilibrium behavior of magnetic filaments made of ellipsoidal particles by means of computer simulations. Our results give evidence that the anisotropy of the particles leads to significant qualitative changes in equilibrium configurations within the range of explored parameters, replacing the structural closure observed for filaments with spherical particles at cooling by monotonic behaviors. We expect these results to be the basis of the future theoretical models and to be valuable for the design of novel magnetic systems.

Acknowledgements. This research was supported by the Ministry of Education and Science of Russia (project no. 3.1438.2017/4.6). P.A.S. acknowledges support of the Austrian Science Fund (FWF):START-Projekt Y 627-N27.

\section{References}

[1] J. Byrom, P. Han, M. Savory, and S.L. Biswal. Directing assembly of DNA-coated colloids with magnetic fields to generate rigid, semiflexible, and flexible chains. Langmuir, vol. 30 (2014), no. 30, pp. 9045-9052.

[2] S. SRivastava, et al. Two-dimensional dna-programmable assembly of nanoparticles at liquid interfaces. Journal of the American Chemical Society, vol. 136 (2014), pp. 8323-8332.

[3] S. Sacanna, L. Rossi, and D.J. Pine. Magnetic click colloidal assembly. J. Am. Chem. Soc., vol. 134 (2012), no. 14, pp. 6112-6115.

[4] A. Perro, et al. Design and synthesis of janus micro- and nanoparticles. J. Mater. Chem., vol. 15 (2005), pp. 3745-3760.

[5] S. Sacanna, L. Rossi, B.W. M. Kuipers, and A.P. Philipse. Fluorescent monodisperse silica ellipsoids for optical rotational diffusion studies. Langmuir, vol. 22 (2006), no. 4, pp. 1822-1827. PMID: 16460113.

[6] P.A. SÁnchez, et al. Supramolecular magnetic brushes: the impact of dipolar interactions on the equilibrium structure. Macromolecules, vol. 48 (2015), no. 20 , pp. $7658-669$.

[7] J.G. Gay and B.J. Berne. Modification of the overlap potential to mimic a linear site-to-site potential. J. Chem. Phys., vol. 74 (1981), pp. 3316-3319. 
Influence of the particle shape on the equilibrium morphologies of supracolloidal...

[8] A. Arnold, B.A. Mann, H. Limbach, and C. Holm. ESPResSo - An Extensible Simulation Package for Research on Soft Matter Systems. In: Forschung und wissenschaftliches Rechnen 2003, vol. 63 of GWDG-Bericht (K. Kremer and V. Macho, Eds, Gesellschaft für wissenschaftliche Datenverarbeitung mbh, Göttingen, Germany, 2004), pp. 43-59.

[9] D.A. Rozhkov, et al. Self-assembly of polymer-like structures of magnetic colloids: Langevin dynamics study of basic topologies. Molecular Simulation, (2017), pp. 1-9 (in press).

[10] S. Kantorovich, E. Pyanzina, and F. Sciortino. The influence of shape anisotropy on the microstructure of magnetic dipolar particles. Soft Matter, vol. 9 (2013), pp. 6594-6603.

Received 27.12.2017 